\title{
十二烷基硫酸钠与甜菜碱在气液和油水界面的复配协同作用研究
}

\author{
李亚娉 ${ }^{a}$ 吕韦钦 ${ }^{a}$ 曹绪龙 ${ }^{b}$ 宋新旺 ${ }^{b}$ 王其伟 ${ }^{b}$ 李英 ${ }^{*, a}$ \\ ( ${ }^{a}$ 山东大学化学与化工学院胶体与界面化学教育部重点实验室 济南 250100) \\ ( ${ }^{b}$ 中石化胜利油田地质科学研究院 东营 257015)
}

\begin{abstract}
摘要 研究了十二烷基硫酸钠(SDS)和甜菜碱(Betaine)以及复配体系在油水界面和气液界面的排布行为, 探讨了温度、 无机盐和复配比例对表面活性剂界面活性和泡沫稳定性的影响, 重点探讨了多价无机阳离子对表面活性剂界面吸附行 为和复配协同加合增效作用的影响，得到了海水为介质条件下两方面性能均较好的体系，取得的认识为高盐条件下低 张力泡沫驱油体系的设计与应用提供理论依据和指导.
\end{abstract}

关键词＼cjkstart界面活性; 泡沫稳定性; 复配协同; 低张力泡沫体系

\section{Study of the Synergistic Effect of Sodium Dodecyl Sulfate and Betaine at the Air/Water and Oil/Water Interfaces}
Li, Yaping ${ }^{a}$
Lv, Weiqin ${ }^{a}$
Cao, Xulong ${ }^{b}$
Song, Xinwang ${ }^{b}$
Wang, Qiwei ${ }^{b}$
Li, Ying ${ }^{*, a}$
( ${ }^{a}$ Key Lab for Colloid and Interface Chemistry of State Education Ministry, Shandong University, Jinan 250100)
( ${ }^{b}$ Geological Scientific Research Institute, Shengli Oilfield, Dongying 257015)

\begin{abstract}
In this paper, the molecular array behavior of anionic surfactant sodium dodecyl sulfate SDS and zwitterionic surfactant Betaine at the air/water and oil/water interfaces were investigated by molecular simulation approaches, which helped to understand the effects of temperature, salts and the proportion of components on the interfacial activity and foam stability of the mixed binary systems, especially how the multivalent inorganic cations influence the interfacial adsorption behavior of the surfactants and the synergistic effect. The oil/water interfacial tension was not only measured experimentally using TEXAS500 spinning drop interface tension meter, but also calculated theoretically using dissipative particle dynamic (DPD) method. Foam decay method was utilized to determine the foam stability. The array behavior of surfactant molecules at the air/water and oil/water interfaces was described by molecular dynamics (MD) simulation method. It was found that the $\mathrm{oil} /$ water interfacial activity of mixed binary system was significantly better than unitary system. The synergistic effect between SDS and Betaine was enhanced when there was $\mathrm{Ca}^{2+}$ or $\mathrm{Mg}^{2+}$ existed in the solution. The radial distribution function of head groups of surfactants around inorganic ions showed that there was very strong interaction between the $\mathrm{Ca}^{2+}$ or $\mathrm{Mg}^{2+}$ and the head groups of SDS and Betaine, which not only induced the increase of the maximum interfacial adsorption quantity of the surfactants, but also adjusted the array states of the surfactant molecules in the interface layer. When the proportion of components of SDS/Betaine binary system was $4: 6$, the oil/water interfacial activity and foam stability of the binary system both were very good with $\mathrm{Ca}^{2+}$ or $\mathrm{Mg}^{2+}$ existing, even use sea water as medium, which could be a good candidate system for foam system used under high salinity condition. The co-adsorption behavior of the surfactant molecules in the binary system at oil/water interface and the foam films was found to be similar. The simulation results agreed well with the experiment results. The knowledge about the microscopic character of the mixtures of surfactants at interfaces could provide useful guidance for the design and application of surfactants under the condition of high salinity, such as low tension foam flooding system used in offshore EOR.
\end{abstract}

Keywords interfacial activity; foam stability; synergistic effect; low tension foam system

\section{1 引言}

表面活性剂由于具有增溶、乳化、润湿、起泡、分 散、去污等功能，在食品、日化、油田、造纸、纺织、 医药等工业领域均具有广泛的应用 ${ }^{[1 \sim 5]}$. 多年来的研究 与实践表明, 单一表面活性剂的功能往往不够理想, 利
用表面活性剂复配可使应用性能得到较大程度的改善, 因此表面活性剂复配协同效应的研究与应用一直很受 关注 ${ }^{[6 \sim 8]}$. 已有的报道中, 关于非离子和离子型表面活 性剂复配体系的研究报道较多, 研究表明非离子表面活 性剂与离子型表面活性剂在界面共吸附，降低极性基间

\footnotetext{
*E-mail: yingli@sdu.edu.cn; Tel.: 0531-88362078
}

Received February 22, 2014; published April 16, 2014.

Project supported by the National Natural Science Foundation of China (No. 21173134) and the National Major Science and Technology Project (No. 2008ZX05011).

项目受国家自然科学基金(No. 21173134)和国家科技重大专项(No. 2008ZX05011)资助. 
的电性斥力, 增大界面吸附量, 提高界面活性的效果十 分显著 ${ }^{[9 \sim 11]}$. 也有报道表明两性表面活性剂与离子型表 面活性剂在混合胶束化和表面吸附中也存在复配协同 作用 ${ }^{[12,13]}$. 比如两性表面活性剂甜菜碱和离子型表面活 性剂十二烷基硫酸钠 SDS 混合体系的复配增效作用在 研究和应用中得到了证实 ${ }^{[14 \sim 16]}$. 两性表面活性剂分子 与非离子表面活性剂一样具有优良的抗盐性, 却没有浊 点的限制, 而且有些两性表面活性剂具有杀菌防霉及抗 静电作用, 还具有良好的生物降解性, 安全无毒无污 染 ${ }^{[17,18]}$. 采用两性表面活性剂与离子型表面活性剂复配 提高体系的抗盐性, 并进一步提高界面活性, 对高盐条 件下表面活性剂的应用具有重要意义.

复配体系中表面活性剂的分子行为和相互作用决 定其性能，在很多应用中界面吸附行为是关键，但由于 对界面研究手段的限制, 对表面活性剂界面分子行为的 信息比较缺乏, 表面活性剂复配增效的微观机制仍不清 晰 ${ }^{[6]}$. 已有研究表明, 无机盐对表面活性剂的性质影响 很大, 特别是多价无机阳离子与表面活性剂间的相互作 用可能极大地影响表面活性剂的行为 ${ }^{[16]}$. 但目前关于 高盐条件对复配表面活性剂分子行为和相互作用的微 观信息的报道较少, 高盐条件下表面活性剂复配增效规 律和微观机制认识的缺乏使得实际应用缺乏必要的依 据和指导.

本文研究两性表面活性剂月桂酰胺基丙基甜菜碱 Betaine 与阴离子型表面活性剂十二烷基硫酸钠 SDS 复 配体系的油水界面活性和泡沫稳定性, 考察温度、无机 盐及复配比例对两方面性能的影响, 发现该两种表面活 性剂具有复配协同加合增效效应，而且该效应在高盐条 件下进一步加强, 其复配体系在海水为介质的情况下具 有较高的界面活性和泡沫稳定性; 采用分子动力学 (MD)模拟方法研究高盐条件下 SDS 和 Betaine 在气液和 油水界面上的分子排布行为, 发现 $\mathrm{Ca}^{2+}$ 和 $\mathrm{Mg}^{2+}$ 与表面 活性剂发生相互作用, 改变了表面活性剂分子的排布行 为, 进而影响了性能. 该研究从分子水平上揭示了无机 离子对表面活性剂分子行为和复配协同作用的影响机 制, 取得的认识为高盐条件下低张力泡沫驱油体系的设 计与应用提供理论依据和指导.

\section{2 结果与讨论}

\subsection{SDS 与 Betaine 及其复配体系在油水界面的排布 行为与界面活性}

图 1(a)曲线是 $7000 \mathrm{mg} / \mathrm{L} \mathrm{NaCl}$ 盐度条件下 $0.2 \mathrm{wt} \%$ 表面活性剂水溶液与正十二烷的界面张力随 SDS 与 Betaine 复配比例的变化曲线, 可以看出, SDS 与 Betaine 单一体系的界面张力均较高, 复配体系的界面张力低于 单一体系, 并且随着 Betaine 比例的增大呈现先降低后 升高的趋势, 在 SDS 与 Betaine 比例(质量比)为 $8: 2 \sim$ $2: 8$ 的较宽范围内界面活性都较高. 对 SDS 与 Betaine
及其复配体系在油水界面排布行为进行了耗散颗粒动 力学(DPD)模拟，图 1(b)曲线是 DPD 模拟计算出的油水 界面张力随 SDS 与 Betaine 复配比例的变化曲线, 趋势 与实验测定结果完全吻合, 复配体系的界面活性强于单 组分体系. 图 1(c)曲线是 DPD 模拟给出的表面活性剂在 油水界面达到饱和吸附时的最大界面吸附量, 从结果可 以看出, 单一 SDS 和 Betaine 体系的最大界面饱和吸附 量均较小, 所有复配体系在表面活性剂分子的界面吸附 达到饱和时的总分子个数均高于单一体系, 且在 SDS 与 Betaine 比例(质量比)为 $2: 8 \sim 4: 6$ 的范围内最大界 面吸附量均较高. 图 1 中沿(c)线的小图是对应体系 DPD 模拟结果的剖面直观图，可以看出 SDS 与 Betaine 分子 共吸附使得界面层内分子排布更均匀致密. 前述结果表 明, SDS 与 Betaine 在油水界面具有协同吸附加合增效作 用. SDS 与 Betaine 比例(质量比)为 $8: 2$ 时界面分子最大 吸附量也较大, 但界面张力未达到最低值, 说明复合体 系的界面效率与配比有关 ${ }^{[19]}$.

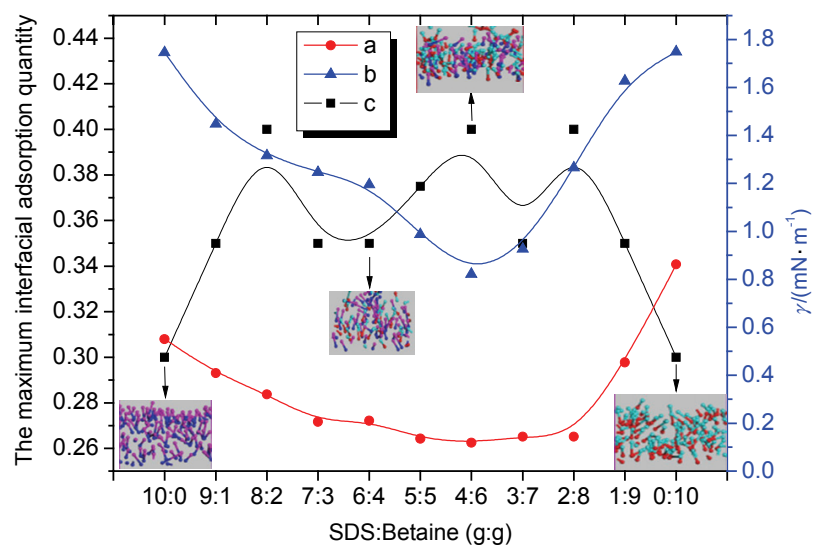

图 $1 \mathrm{SDS}$ 与 Betaine 及其复配体系的十二烷/水界面张力及表面活性 剂界面饱和吸附量随复配比例的变化曲线. (a) 实验测定的界面张力结 果 $\left(7000 \mathrm{mg} / \mathrm{L} \mathrm{NaCl}, C_{\text {总 }}=0.2 \mathrm{wt} \%\right.$ ); (b) DPD 模拟的界面张力结果; (c) DPD 模拟给出的最大界面吸附量. 沿(c)线的小图是箭头起点对应体 系的 DPD 模拟结果的剖面直观图, 略去水分子和油分子, 珠子设定 为: 玫红色, SDS 亲水头基; 深蓝色, SDS 疏水尾链; 亮蓝色, Betaine 亲水头基; 红色, Betaine 疏水尾链.

Figure 1 Variation of dodecane/water interfacial tension and the maximum interfacial adsorption quantity of SDS/Betaine binary system as a function of the component ratio. (a) experimental interfacial tension $\left(7000 \mathrm{mg} / \mathrm{L} \mathrm{NaCl}, C_{\text {total }}=0.2 \mathrm{wt} \%\right)$; (b) interfacial tension in DPD simulation; (c) the maximum interfacial adsorption quantity in DPD simulation. Small pictures along curve (c) are section illustrative diagrams of DPD simulation corresponding to the system at the starting point of the arrow, omit water and oil, beads are set as: rose red, head group of SDS; mazarine, tail chain of SDS; bright blue, head group of Betaine; red, tail chain of Betaine

图 2 所示为表面活性剂总浓度保持为 $0.2 \mathrm{wt} \%$ 时不 同配比复配体系的油水界面张力随温度的变化. 从图中 结果可以看出, 在考察的温度范围内 $\left(25 \sim 55{ }^{\circ} \mathrm{C}\right)$, 单一 SDS 体系的界面活性随温度的升高而降低, 而 Betaine 的油水界面活性随温度升高而增强，二者的复配体系的 油水界面张力均低于单一体系, SDS 与 Betaine 的比例 
(质量比)为 $7: 3 \sim 2: 8$ 的复配体系界面张力降低的程 度均较大, 其中比例为 $4: 6$ 的复配体系在不同温度时 油水界面张力均较低, 是较优配比.

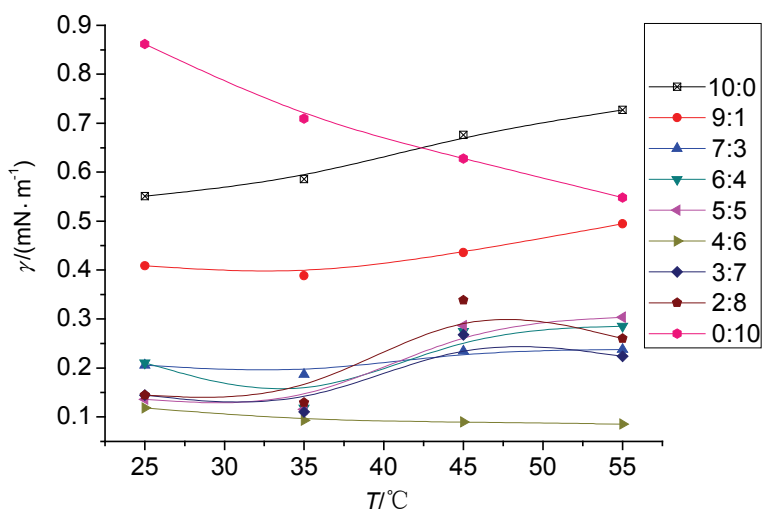

图 $27000 \mathrm{mg} / \mathrm{L} \mathrm{NaCl}$ 盐度下不同配比的 SDS 与 Betaine 混合体系水 溶液与十二烷的界面张力值随温度的变化. 表面活性剂总浓度为 0.2 wt $\%$

Figure 2 Variation of the interfacial tension of solutions of SDS/Betaine binary system containing $7000 \mathrm{mg} / \mathrm{L} \mathrm{NaCl}$ with dodecane as a function of temperature. The total concentration of surfactants is 0.2 wt $\%$

\section{2 无机盐对 SDS 与 Betaine 及其复配体系油水界面 活性和分子行为的影响}

图 3 所示是不同的无机盐溶液中, 表面活性剂总浓 度均为 $0.2 \mathrm{wt} \%$ 的情况下, SDS 与 Betaine 及其复配体系 的水溶液与十二烷在 $55{ }^{\circ} \mathrm{C}$ 下的油水界面张力值, 无机 盐浓度参照海水组成. 从图中可以看出, 在几种不同的 无机盐溶液中, 复配体系的界面活性也都高于单一体 系. 特别值得注意的是, 在含有 $\mathrm{Ca}^{2+}$ 的介质环境中单一 组分和复配体系的界面活性均极大地增强, 复配体系在 很宽的配比范围内界面张力值均达到了 $10^{-2} \mathrm{mN} / \mathrm{m}$ 量 级, 说明该体系很适用于含钲离子的矿化水环境. 对于 SDS 或 Betaine 单一组分体系, 含有 $\mathrm{MgCl}_{2}$ 的体系的界 面张力值比含有 $\mathrm{NaCl}$ 体系的界面张力值高, 但对于复 配体系两种介质环境下的界面张力则很相近.

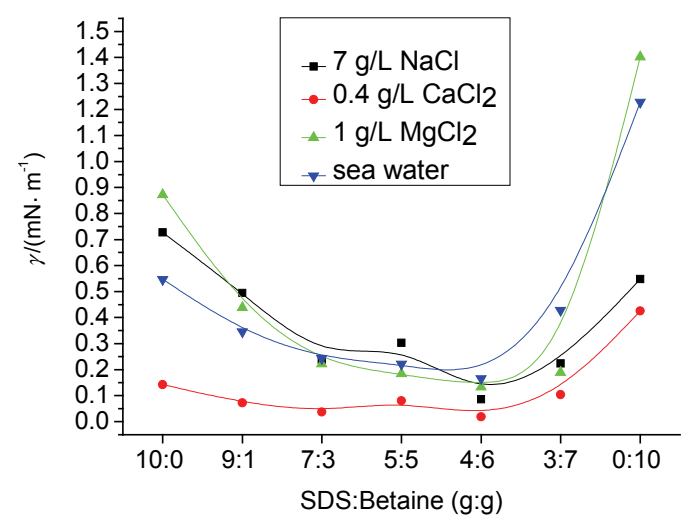

图 $355{ }^{\circ} \mathrm{C}$ 下含不同无机盐的表面活性剂溶液与十二烷的界面张力 值随复配比例的变化

Figure 3 Variation of interfacial tension of mixed systems of surfactants with different inorganic salts and dodecyl as a function of the component ratio at $55{ }^{\circ} \mathrm{C}$
为了深入了解无机离子对表面活性剂界面行为的 影响, 采用分子动力学(MD)模拟方法研究油水界面表 面活性剂分子的微观行为. 表 1 中列出了水溶液中分别 含有 $\mathrm{Na}^{+} 、 \mathrm{Ca}^{2+}$ 和 $\mathrm{Mg}^{2+}$ 时表面活性剂复配体系的界面 生成能, 含 $\mathrm{Na}^{+}$和含 $\mathrm{Mg}^{2+}$ 体系的界面生成能相差不多, 而含 $\mathrm{Ca}^{2+}$ 的体系的界面生成能则明显低于含 $\mathrm{Na}^{+}$和 $\mathrm{Mg}^{2+}$ 的体系, 这与图 3 中的实验结果一致.

表 $1 \mathrm{SDS} /$ Betaine/正十二烷/水体系分别含 $\mathrm{Na}^{+}, \mathrm{Ca}^{2+}$ 和 $\mathrm{Mg}^{2+}$ 时的界 面生成能

Table 1 Interfacial formation energy of the SDS/Betaine/dodecyl/water system containing $\mathrm{Na}^{+}, \mathrm{Ca}^{2+}$ or $\mathrm{Mg}^{2+}$

\begin{tabular}{ccc}
\hline System & $n^{a}$ & $\mathrm{IFE} /\left(\mathrm{kJ} \cdot \mathrm{mol}^{-1}\right)$ \\
\hline $\mathrm{Na}^{+}$ & 44 & -191.2 \\
$\mathrm{Mg}^{2+}$ & 44 & -195.6 \\
$\mathrm{Ca}^{2+}$ & 44 & -228.9 \\
\hline
\end{tabular}

${ }^{a} n$ the number of surfactant molecules. $n_{\mathrm{SDS}}: n_{\text {Betaine }}=10: 12 . \quad n_{\mathrm{Na}+}=480$; $n_{\mathrm{Ca} 2+}=16 ; n_{\mathrm{Mg} 2+}=64$.

图 4 是水溶液中分别含有 $\mathrm{Ca}^{2+}$ 和 $\mathrm{Mg}^{2+}$ 时复配表面 活性剂 $\left(n_{\mathrm{SDS}}: n_{\text {Betaine }}=10: 12\right)$ 在油水界面排布的模拟 直观图，由此分析各分子基团和离子的空间分布. 分别 统计硫酸头基和羧酸基周围 $\mathrm{Mg}^{2+}$ 与 $\mathrm{Ca}^{2+}$ 的径向分布函 数, 结果见图 5. 从图中可以看出, 各分布函数均在 $5 \AA$ 范围内出现两个峰, 表明 $\mathrm{Mg}^{2+}$ 与 $\mathrm{Ca}^{2+}$ 均与 SDS 和 Betaine 有强相互作用. 相比而言, $-\mathrm{COO}^{-}$与 $\mathrm{Ca}^{2+}$ 的相互 作用比 $\mathrm{Mg}^{2+}$ 强, $-\mathrm{SO}_{4}{ }^{-}$与 $\mathrm{Mg}^{2+}$ 和 $\mathrm{Ca}^{2+}$ 的相互作用均较 强. 界面层内 $\mathrm{Ca}^{2+}$ 周围- $\mathrm{COO}^{-}$分布明显高于 $-\mathrm{SO}_{4}^{-}, \mathrm{Mg}^{2+}$ 周围- $-\mathrm{SO}_{4}{ }_{4}^{-}$的分布略高于- $\mathrm{COO}^{-}$. 由此可知, $\mathrm{SDS}$ 极性基 与 $\mathrm{Mg}^{2+}$ 和 $\mathrm{Ca}^{2+}$ 的相互作用均较强, 而 Betaine 极性基与 $\mathrm{Ca}^{2+}$ 相互作用更强. 在海水中, $\mathrm{Mg}^{2+}$ 的含量远高于 $\mathrm{Ca}^{2+}$, 显然 $\mathrm{Mg}^{2+}$ 与表面活性剂的作用占主导，因此海水基溶 液与油相间的界面张力值与仅含 $\mathrm{Mg}^{2+}$ 时十分相近, 与 图 3 中实验结果相吻合.

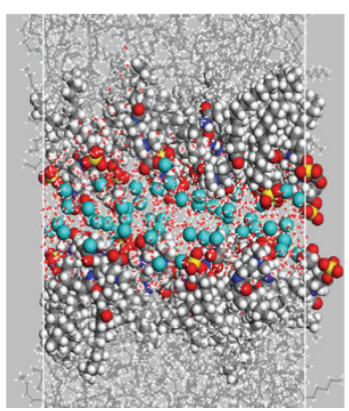

a

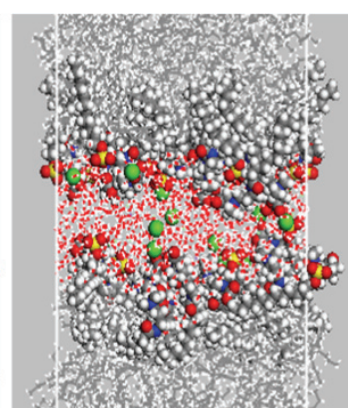

b
图 4 油水界面的分子动力学模拟直观图. SDS 与 Betaine 分子个数比 为 $10: 12$, 质量比均为 $4: 6$. (a)含 $\mathrm{Mg}^{2+}$ 体系 $\left(n_{\mathrm{Mg}^{2+}}{ }^{2}=64\right)$, (b)含 $\mathrm{Ca}^{2+}$ 体 系 $\left(n_{\mathrm{Ca}^{2+}}=16\right)$. 原子或离子标示如下: $\mathrm{Mg}^{2+}$, 蓝色; $\mathrm{Ca}^{2+}$, 绿色; $\mathrm{S}$, 黄 色; $\mathrm{O}$, 红色; $\mathrm{N}$, 深蓝色; $\mathrm{C}$, 灰色; H, 白色

Figure 4 Molecular dynamics simulative illustrative diagram of molecules at oil-water interface. Molecular number proportion of SDS and Betaine is $10: 12$, mass ratio is $4: 6$. (a) with $\mathrm{Mg}^{2+}\left(n_{\mathrm{Mg}^{2+}}{ }^{2}=64\right)$, (b) with $\mathrm{Ca}^{2+}\left(n_{\mathrm{Ca}^{2+}}{ }^{2}=16\right)$. Atoms or ions are labeled as follows: $\mathrm{Mg}^{2+}$, blue; $\mathrm{Ca}^{2+}$, green; $\mathrm{S}$, yellow; $\mathrm{O}$, red; $\mathrm{N}$, mazarine; $\mathrm{C}$, grey; $\mathrm{H}$, white 

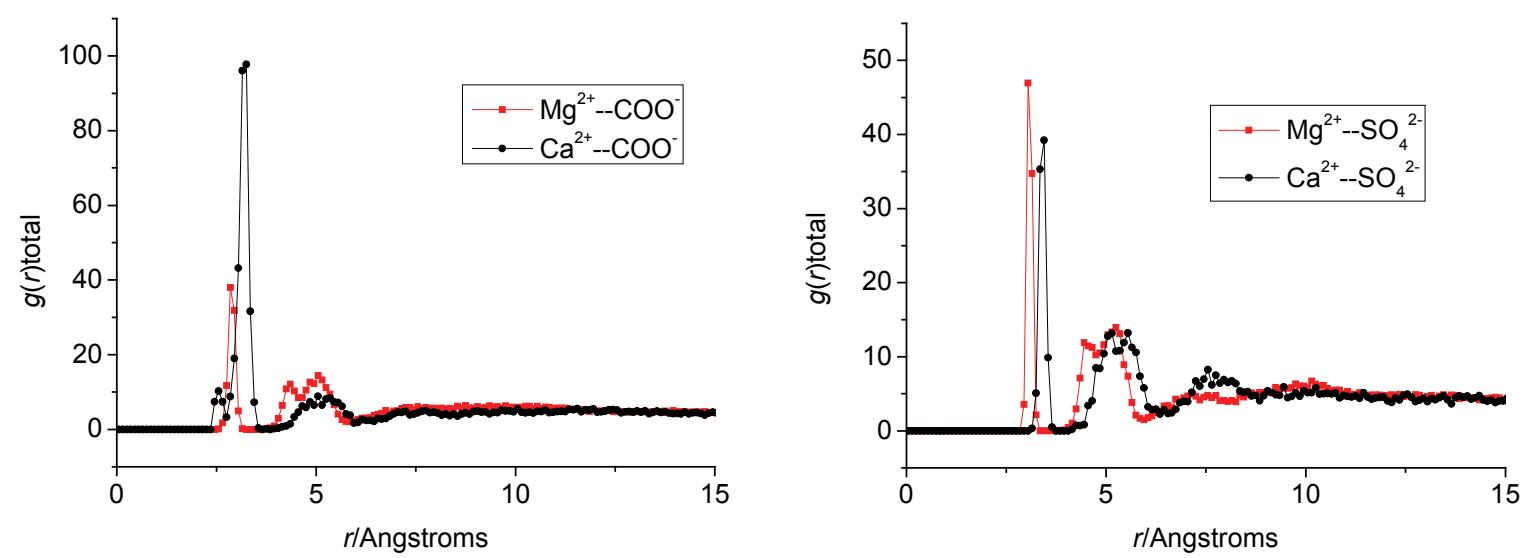

图 5 表面活性剂头基周围无机盐离子的径向分布函数

Figure 5 Radial distribution function of inorganic ions around head groups of surfactants

\subsection{SDS 与 Betaine 及其复配体系在气液界面的分子 行为以及泡沫稳定性}

以 $7000 \mathrm{mg} / \mathrm{L} \mathrm{NaCl}$ 溶液为介质, 配制 $\mathrm{SDS}$ 与 Betaine 单一和复配体系的溶液, 总浓度均为 $0.2 \mathrm{wt} \%$, 分别在恒定温度为 $30{ }^{\circ} \mathrm{C}, 40{ }^{\circ} \mathrm{C}, 50{ }^{\circ} \mathrm{C}, 60{ }^{\circ} \mathrm{C}$ 下测定泡 沫衰减曲线, 结果见图 6. 从图中结果可以看出在较低 温度 $\left(30{ }^{\circ} \mathrm{C}, 40{ }^{\circ} \mathrm{C}\right)$ 下, 复配体系的泡沫稳定性优于单
一表面活性剂体系. 随着温度升高, 单一 SDS 体系的泡 沫稳定性明显下降, 温度每升高 $10{ }^{\circ} \mathrm{C}$, 半衰期的时间 大约降低一倍; 单一 Betaine 体系的泡沫稳定性受温度 的影响很小, 随着温度升高, 半衰期几乎不变. 在温度 高于 $50{ }^{\circ} \mathrm{C}$ 的情况下, SDS 与 Betaine 复配体系的泡沫稳 定性比单一SDS 好, 但是低于单一Betaine 体系.
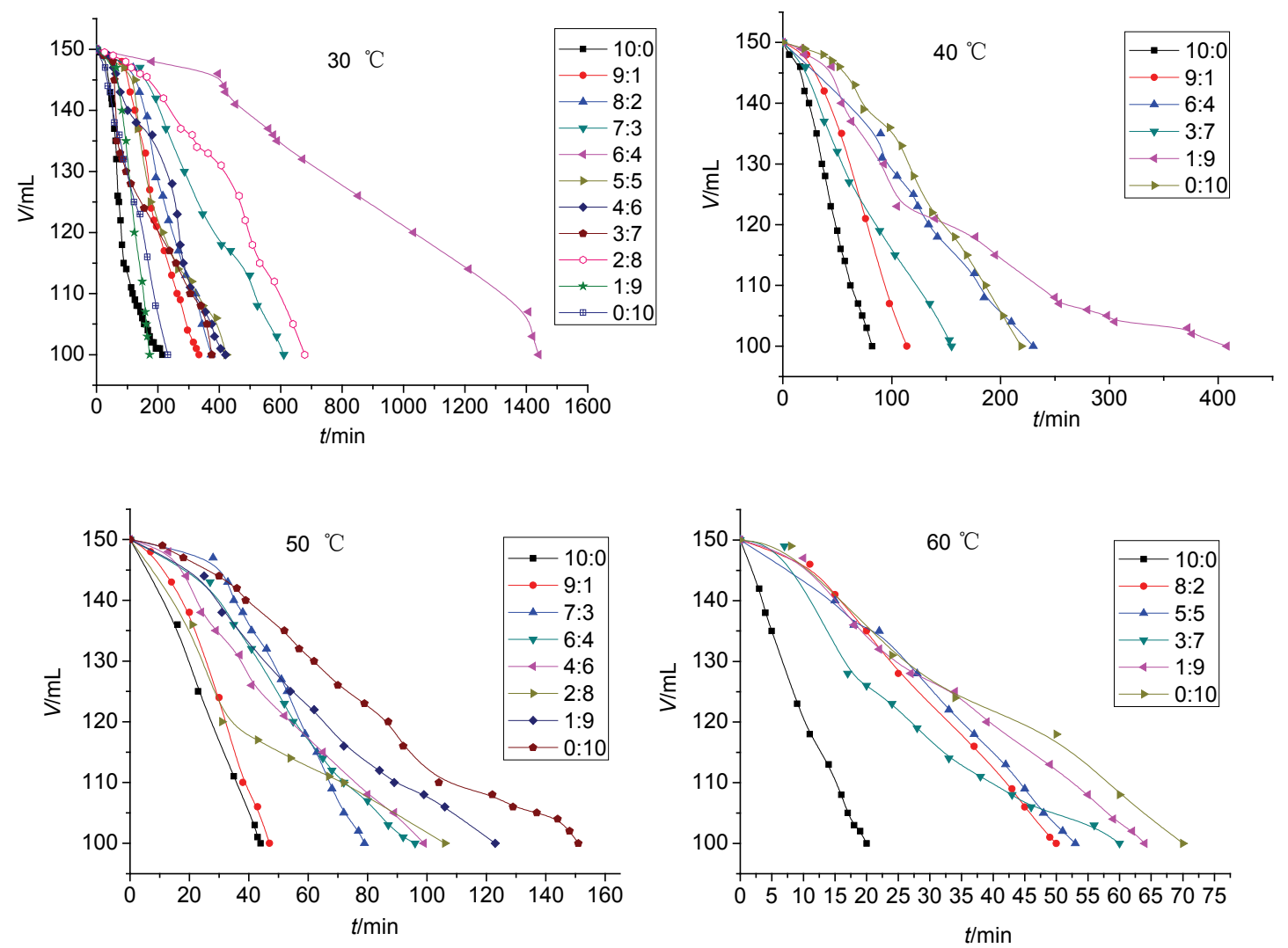

图 6 不同温度下 SDS 与 Betaine 以及不同比例复配体系的泡沫衰减曲线. 表面活性剂总浓度均为 $0.2 \mathrm{wt} \%$, 以 $7000 \mathrm{mg} / \mathrm{L} \mathrm{NaCl}$ 溶液为介质.

Figure 6 Foam decay curves of SDS, Betaine and mixed systems at different temperatures. Total concentration of surfactant is $0.2 \mathrm{wt} \%$, put $7000 \mathrm{mg} / \mathrm{L}$ $\mathrm{NaCl}$ solution as medium 
图 7 所示是 $60{ }^{\circ} \mathrm{C}$ 下分别由 SDS 和 Betaine 稳定的 泡沫液膜的 MD 模拟直观图. 从图中看出, SDS 分子在 界面上发生了聚集, 不能很好地分散排布于界面, 界面 覆盖程度低, 因此泡沫稳定性下降; 而相同温度条件下, 泡沫液膜内 Betaine 分子的聚集趋势不明显, 在气液界 面上的分布相对比较均匀, 对于泡沫的稳定起到较好的 作用. 因此在较高的温度下, Betaine 对泡沫的稳定能力 比 SDS 高.

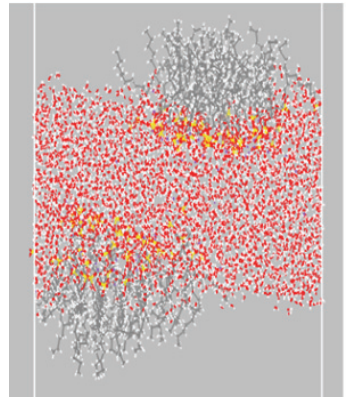

a

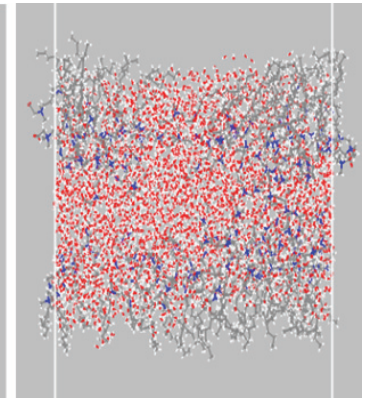

$\mathrm{b}$
图 $760{ }^{\circ} \mathrm{C}$ 下 $\operatorname{SDS}(\mathrm{a})$, Betaine (b)稳定的泡沫液膜的模拟直观图. $n=$ 25 , 原子标示如下: $\mathrm{S}$, 黄色; $\mathrm{O}$, 红色; $\mathrm{N}$, 深蓝色; $\mathrm{C}$, 灰色; $\mathrm{H}$, 白色 Figure 7 Simulative illustrative diagram of foam liquid film stabilized by SDS (a), Betaine (b) at $60{ }^{\circ} \mathrm{C} . n=25$, atoms are labeled as follows: $\mathrm{S}$, yellow; $\mathrm{O}$, red; $\mathrm{N}$, mazarine; $\mathrm{C}$, grey; $\mathrm{H}$, white

\section{4 无机盐对 SDS 与 Betaine 及其复配体系泡沫稳定 性的影响}

用含不同无机盐的溶液为介质配制 SDS 与 Betaine 及复配体系的溶液, 总浓度为 $0.2 \mathrm{wt} \%$, 测定泡沫衰减 曲线. 图 8 示出了 $50{ }^{\circ} \mathrm{C}$ 下不同配比体系的泡沫半衰期. 从图中看出, 含有 $\mathrm{Ca}^{2+}$ 或 $\mathrm{Mg}^{2+}$ 的体系的泡沫稳定性均 远远高于含 $\mathrm{Na}^{+}$的情况, 显然在含有 $\mathrm{Ca}^{2+}$ 或 $\mathrm{Mg}^{2+}$ 的情 况下，体系体现出更显著的复配增效作用.

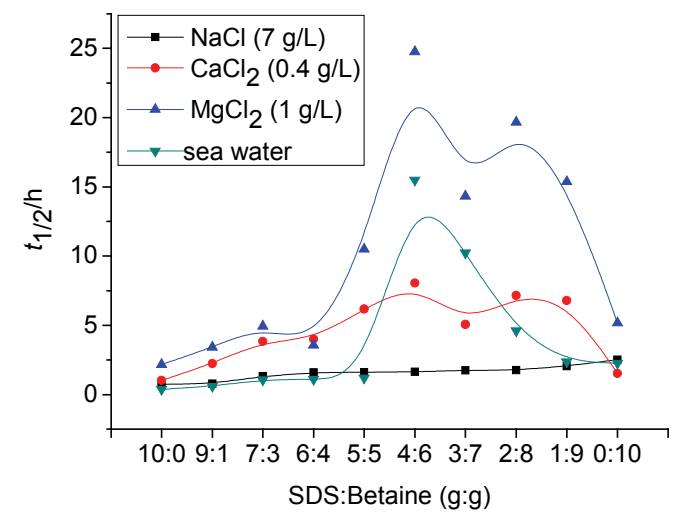

图 $850{ }^{\circ} \mathrm{C}$ 下采用不同无机盐溶液为介质的不同复配比例的复配体 系溶液形成的泡沫的半衰期. 表面活性剂总浓度均为 $0.2 \mathrm{wt} \%$

Figure 8 The half life time of the foams formed from solutions of mixed surfactant systems with different component ratios containing different inorganic salts at $50{ }^{\circ} \mathrm{C}$. Total concentration of surfactant is 0.2 wt $\%$
图 9 所示是 SDS 与 Betaine 复配体系的泡沫液膜的 动力学模拟直观图. 分析体系中基团和离子的分布, 统 计表面活性剂极性基周围 $\mathrm{Ca}^{2+}$ 和 $\mathrm{Mg}^{2+}$ 的径向分布函数, 结果见图 10. 图 10 的结果与图 5 非常相似, 表明气液界 面和油水界面表面活性剂分子行为有较高的相似性, $\mathrm{Mg}^{2+}$ 和 $\mathrm{Ca}^{2+}$ 均与 SDS 和 Betaine 有显著的相互作用.

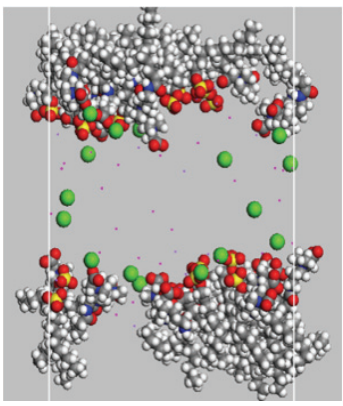

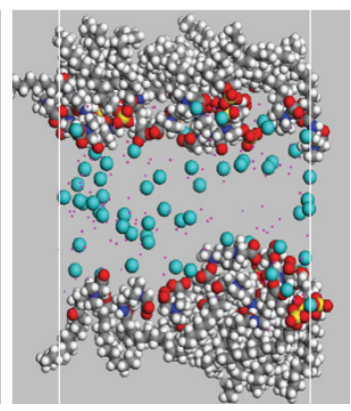

b
图 9 泡沫液膜的动力学模拟直观图. 略去水分子. SDS 与 Betaine 分 子个数比为 $10: 12$, 质量比均为 $4: 6$. (a)含 $\mathrm{Ca}^{2+}$ 体系 $\left(n_{\mathrm{Ca}^{2+}}{ }^{2+}=16\right)$, (b)含 $\mathrm{Mg}^{2+}$ 体系 $\left(n_{\mathrm{Mg}^{2+}}=64\right)$. 图中原子或离子标示如下: $\mathrm{Ca}^{2+}$, 绿色; $\mathrm{Mg}^{2+}$, 蓝色; $\mathrm{S}$, 黄色; $\mathrm{O}$, 红色; $\mathrm{Na}^{+}$, 紫色; $\mathrm{Cl}^{-}$, 玫红色; $\mathrm{N}$, 深蓝色; $\mathrm{C}$, 灰色; H, 白色

Figure 9 Molecular dynamics simulative illustrative diagram of foam liquid film. Omit water molecules. Molecular number proportion of SDS and Betaine is $10: 12$, mass ratio is $4: 6$. (a) with $\mathrm{Ca}^{2+}\left(n_{\mathrm{Ca}^{2}}{ }^{2+}=16\right)$, (b) with $\mathrm{Mg}^{2+}\left(n_{\mathrm{Mg}^{2+}}=64\right)$. Atoms or ions are labeled as follows: $\mathrm{Ca}^{2+}$, green; $\mathrm{Mg}^{2+}$, blue; $\mathrm{Na}^{+}$, purple; $\mathrm{Cl}^{-}$, rose red; $\mathrm{S}$, yellow; $\mathrm{O}$, red; $\mathrm{N}$, mazarine; $\mathrm{C}$, grey; $\mathrm{H}$, white
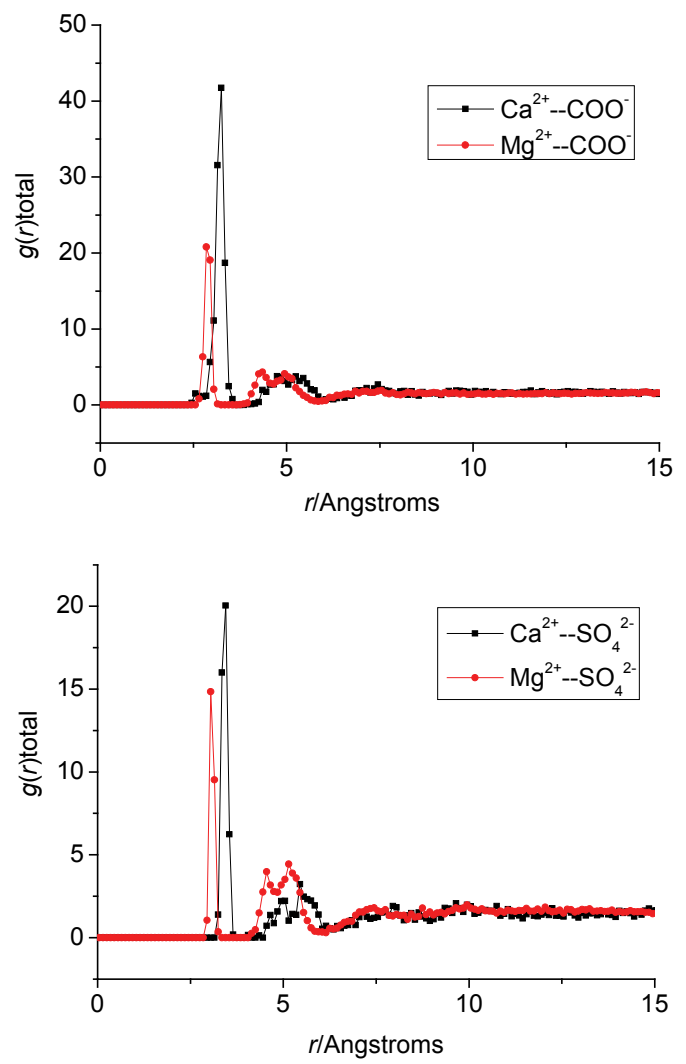

图 10 表面活性剂头基周围无机盐离子的径向分布函数

Figure 10 Radial distribution function of inorganic ions around head group of surfactants 

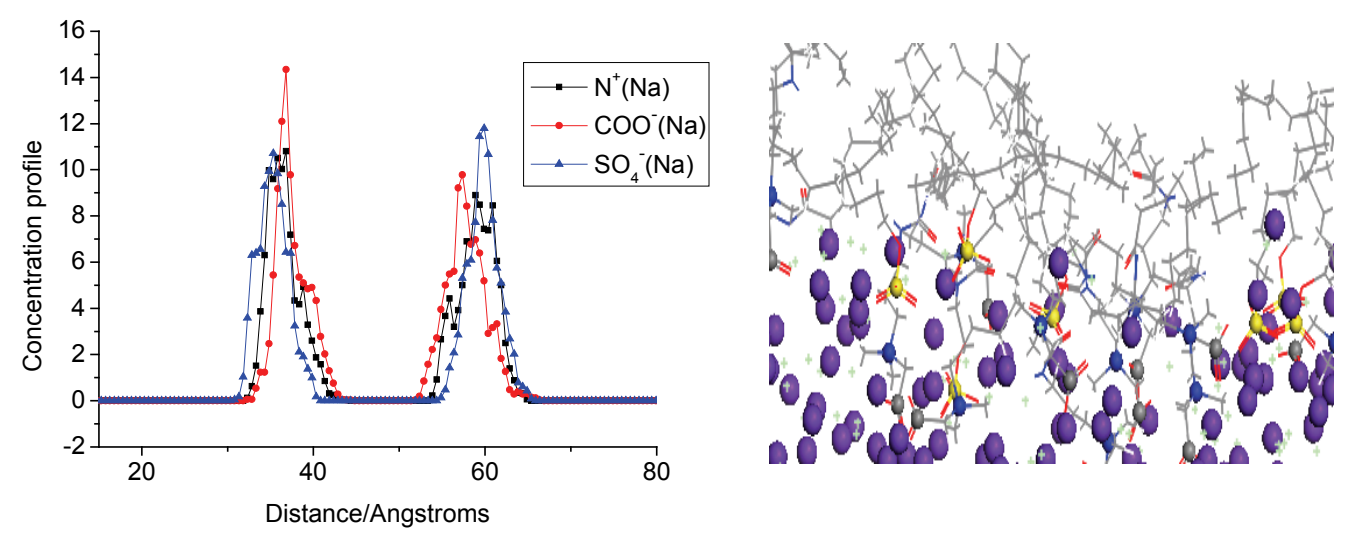

a
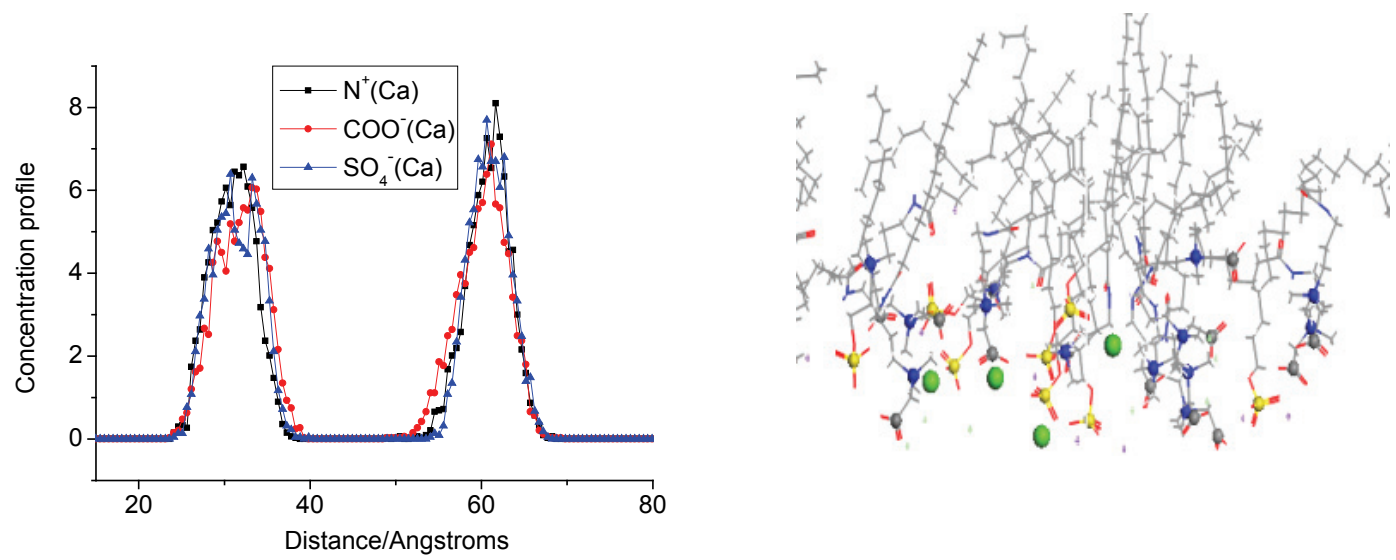

b
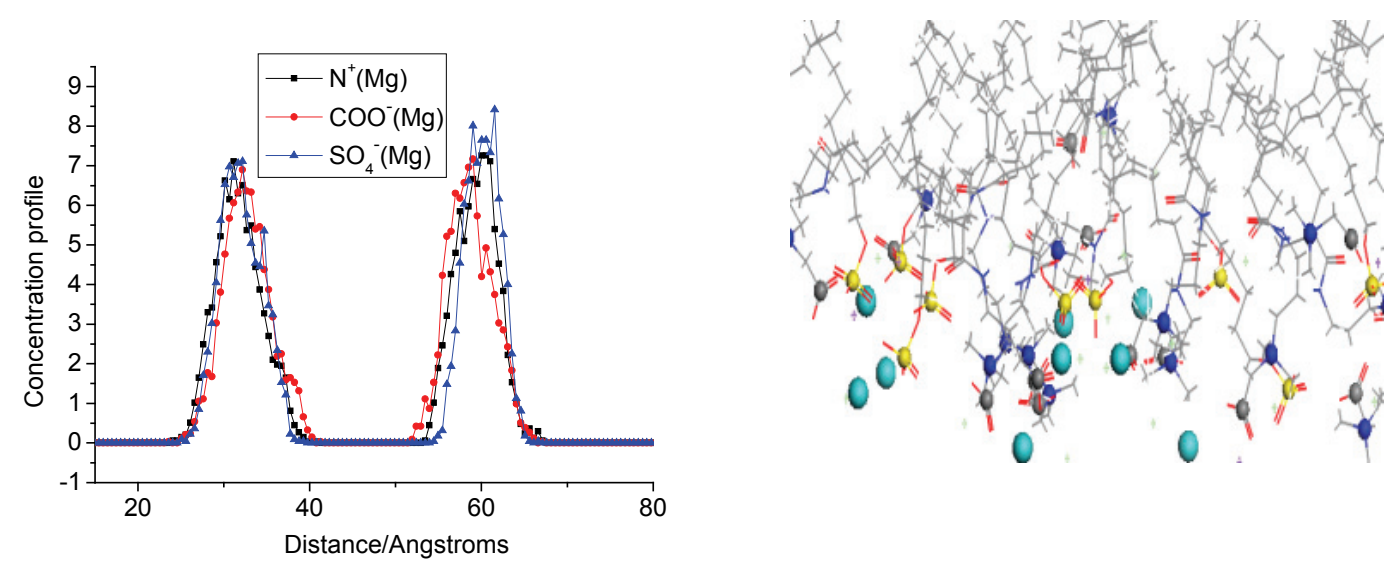

图 11 各体系中表面活性剂头基的浓度分布图及对应的界面层分子直观图. (a)含 $\mathrm{Na}^{+}$体系 $\left(n_{\mathrm{Na}+}=480\right),(\mathrm{b})$ 含 $\mathrm{Ca}^{2+}$ 体系 $\left(n_{\mathrm{Ca} 2+}=16\right),(\mathrm{c})$ 含 $\mathrm{Mg}^{2+}$ 体系 $\left(n_{\mathrm{Mg} 2}=64\right)$. 图中原子或离子标示如下: $\mathrm{Na}^{+}$, 紫色; $\mathrm{Ca}^{2+}$, 绿色; $\mathrm{Mg}^{2+}$, 蓝色; $\mathrm{S}$, 黄色; $\mathrm{O}$, 红色; $\mathrm{N}$, 深蓝色; $\mathrm{C}$, 灰色

Figure 11 The concentration distribution of the head groups of surfactants and different corresponding illustrative diagrams of interface layer molecules in different systems. (a) with $\mathrm{Na}^{+}\left(n_{\mathrm{Na}+}=480\right)$, (b) with $\mathrm{Ca}^{2+}\left(n_{\mathrm{Ca} 2+}=16\right)$, (c) with $\mathrm{Mg}^{2+}\left(n_{\mathrm{Mg} 2^{+}}=64\right)$. Atoms or ions are labeled as follows: $\mathrm{Na}^{+}$, purple; $\mathrm{Ca}^{2+}$, green; $\mathrm{Mg}^{2+}$, blue; $\mathrm{S}$, yellow; $\mathrm{O}$, red; $\mathrm{N}$, mazarine; $\mathrm{C}$, grey

复配表面活性剂分子在泡沫液膜上垂直于界面方 向的浓度分布见图 11. 由图可见, 在只有 $\mathrm{Na}^{+}$的情况下, SDS 和 Betaine 分子错层排布, SDS 的硫酸基恰好与 Betaine 极性头上的季胺基团(以 $\mathrm{N}$ 原子标记)平齐, 以减
少极性基间的电性斥力，而在 $\mathrm{Ca}^{2+} 、 \mathrm{Mg}^{2+}$ 存在的情况下， SDS 的硫酸基和 Betaine 的羧酸基分布变得平齐, 充分 证明 $\mathrm{Ca}^{2+} 、 \mathrm{Mg}^{2+}$ 进入界面层内，与极性基间具有强相互 作用, 中和了极性基电荷, 因此影响了表面活性剂的界 
面排布，在垂直于界面的方向上极性基分布更平齐，而 且每个多价无机阳离子可以与不止一个表面活性剂分 子发生相互作用，使得表面活性剂的排布更致密，界面 吸附量增大, 这是该表面活性剂复配体系的界面活性与 泡沫稳定性在 $\mathrm{Ca}^{2+} 、 \mathrm{Mg}^{2+}$ 存在时都得到显著增强的重 要原因; 该相互作用也降低了表面活性剂的亲水性, 使 得表面活性剂疏水链的有序性降低. 统计不同情况下表 面活性剂分子尾链相对于界面的倾角分布, 结果见图 12. 由图可以看出, 溶液中只含有 $\mathrm{Na}^{+}$时, SDS 尾链相对 $\mathrm{Z}$ 方向的倾角最大分布在 $60^{\circ}$ 左右, Betaine 尾链相对 $\mathrm{Z}$ 方向的倾角最大分布在 $90^{\circ}$. 镁离子存在时, 导致了 SDS 和 Betaine 尾链倾角分布均变宽, 说明分子尾链的 排布有序性降低, 尾链交错形成网络结构, 有利于降低
气体透过液膜的扩散, 对提高泡沫稳定性有利 ${ }^{[20]}$. 含有 钙离子时, Betaine 的尾链倾角变小, 分布变宽, SDS 尾 链倾角最大分布角度变大至 $75^{\circ}$, 但角度分布变窄. 相 比而言, 含 $\mathrm{Mg}^{2+}$ 的体系的泡沫稳定性比含 $\mathrm{Ca}^{2+}$ 的更高.

以海水为介质时, 泡沫稳定性随表面活性剂配比的 变化规律如图 8 所示. 分析钙、镁离子共存情况下, 表 面活性剂头基周围 $\mathrm{Ca}^{2+}$ 和 $\mathrm{Mg}^{2+}$ 的分布情况, 结果见图 13, 由图看出虽然泡沫液膜上 Betaine 极性基周围仍有 $\mathrm{Ca}^{2+}$ 存在, 但 SDS 极性基周围 $5 \AA$ 范围内只有 $\mathrm{Mg}^{2+}$ 而 没有 $\mathrm{Ca}^{2+}$, 说明在此条件下 $\mathrm{Mg}^{2+}$ 与表面活性剂极性基 的结合占主导; 在海水中, $\mathrm{Mg}^{2+}$ 的含量远高于 $\mathrm{Ca}^{2+}$, 因 此也就不难理解海水为介质的情况下, 泡沫半衰期与仅 含 $\mathrm{Mg}^{2+}$ 的体系接近.
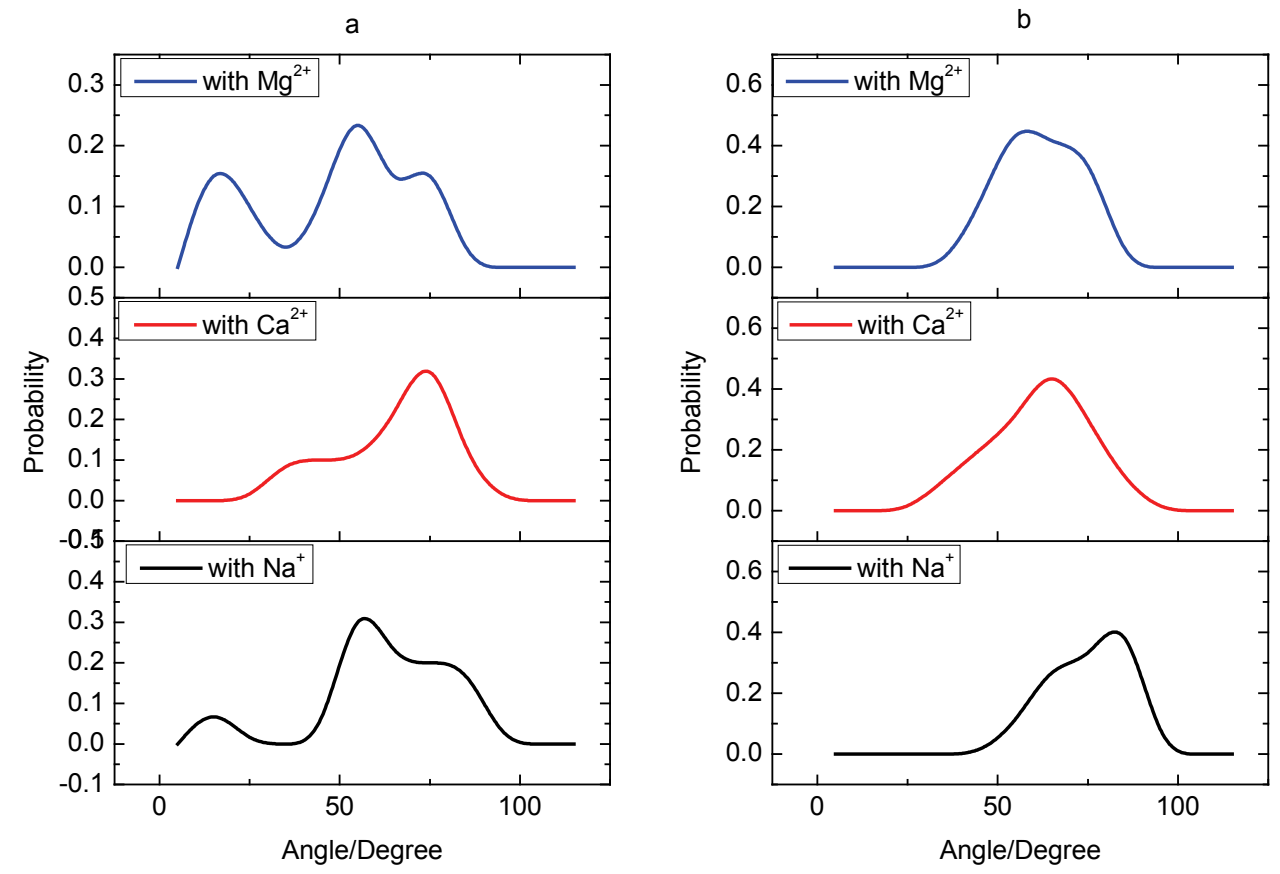

图 12 表面活性剂 SDS (a)和 Betaine (b) 尾链相对 Z 方向倾角分布

Figure 12 Tilt angle orientation distributions with respect to the $Z$ direction of the surfactant hydrophobic tail for (a) SDS and (b) Betaine
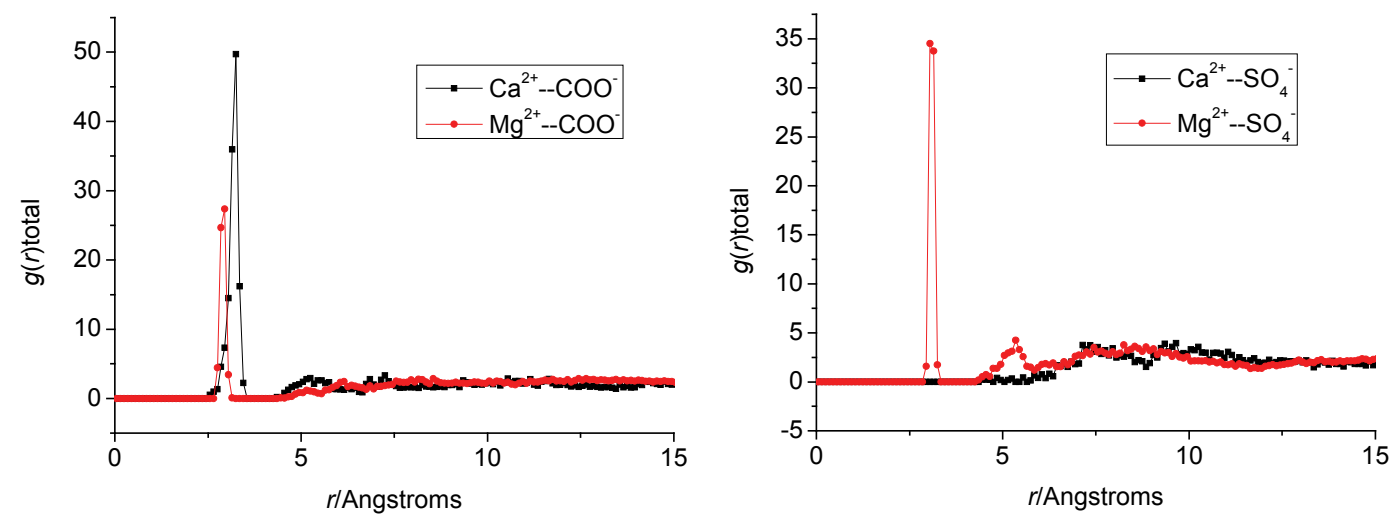

图 13 海水体系中表面活性剂头基周围无机盐离子的径向分布函数

Figure 13 Radial distribution function of inorganic ions around head groups of surfactants in sea water 


\section{3 结论}

通过研究两性表面活性剂月桂酰胺基丙基甜菜碱 Betaine 与阴离子型表面活性剂十二烷基硫酸钠 SDS 复 配体系的油水界面活性和泡沫稳定性, 考察温度、无机 盐及复配比例对两方面性能的影响, 发现二者在较大的 比例范围内均存在复配增效作用, 且该复配增效作用在 有 $\mathrm{Ca}^{2+}$ 和 $\mathrm{Mg}^{2+}$ 时得到进一步加强, 因此适合在高盐条 件下应用. 在含有 $\mathrm{Ca}^{2+}$ 或 $\mathrm{Mg}^{2+}$ 时, SDS 与 Betaine 复配 比例为 $4: 6$ 时加合增效作用较其他复配比例更好, 该 复配体系在海水为介质的情况下同时具有较高的界面 活性和泡沫稳定性.

采用分子动力学 (MD) 模拟方法描述高盐条件下 SDS 和 Betaine 在气液和油水界面上的分子排布行为, 发现 $\mathrm{Ca}^{2+}$ 和 $\mathrm{Mg}^{2+}$ 与表面活性剂极性基发生相互作用, 改变了表面活性剂分子的排布行为, 从而影响了性能; 在海水为介质的情况下, $\mathrm{Ca}^{2+}$ 和 $\mathrm{Mg}^{2+}$ 共存, $\mathrm{Mg}^{2+}$ 的影响 占主导.

该研究从油水和气液界面两个方面出发, 从分子水 平上揭示无机离子对表面活性剂分子行为和复配协同 作用的影响, 取得的认识为高盐条件下低张力泡沫驱油 体系的设计与应用提供理论依据和指导.

\section{4 实验部分}

\section{1 实验试剂}

十二烷基硫酸钠(SDS), 纯度 $>99.0 \%$, Cibio Biotechnology Ltd; 月桂酰胺基丙基甜菜碱(Betaine), 胜利 油田地质科学研究院提供; $\mathrm{NaCl}$ (A.R.), 天津市广成化 学试剂有限公司; 无水 $\mathrm{CaCl}_{2}$ (A.R.), 天津市风船化学 试剂科技有限公司; 正十二烷(A.R.), 天津市科密欧化 学试剂有限公司. 海水为黄海海域海水, 取自日照近海, 经测定总矿化度为 $32082 \mathrm{mg} / \mathrm{L}\left(\mathrm{Na}^{+}: 10638 \mathrm{mg} / \mathrm{L} ; \mathrm{Ca}^{2+}\right.$ : $\left.398 \mathrm{mg} / \mathrm{L} ; \mathrm{Mg}^{2+}: 1042 \mathrm{mg} / \mathrm{L}\right)$. 表面活性剂分子结构如图 14 所示.

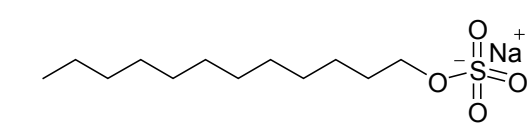

a

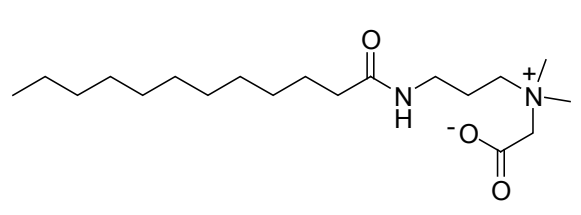

b

图 14 文章中使用的表面活性剂的分子结构示意图

Figure 14 Molecular structures of surfactants used in the paper (a) SDS; (b) Betaine

\section{2 实验研究方法}

\subsection{1 泡沫稳定性测定}

采用泡沫衰减法考察泡沫的稳定性, 直径为 100 $\mu \mathrm{m}$ 的玻璃珠烧结而成的玻璃砂滤板上方预置 $50 \mathrm{~mL}$ 的 测试溶液，以 $200 \mathrm{~mL} / \mathrm{min}$ 的速度从玻璃砂滤板下方注 入氮气，形成一定体积的泡沫柱，观察泡沫的衰减，用 泡沫衰减曲线和泡沫半衰期来衡量泡沫的稳定性 ${ }^{[20 ~ 24]}$.

\section{2 .2 油水界面张力测定}

采用 TEXAS500 旋滴界面张力仪测定油水界面张 力. 油相为正十二烷. 温度分别为 $25 \pm 0.1{ }^{\circ} \mathrm{C}, 35 \pm 0.1$ ${ }^{\circ} \mathrm{C}, 45 \pm 0.1{ }^{\circ} \mathrm{C}, 55 \pm 0.1{ }^{\circ} \mathrm{C}$.

\section{3 分子模拟方法}

\subsection{1 选用的分子模拟软件}

本研究工作中所有的分子模拟工作均采用美国 Accelrys 公司的 Material Studio 5.0 软件计算完成.

\subsection{2 耗散颗粒动力学模拟}

采用 Hoogerbrugge 和 Koelman ${ }^{[25]}$ 提出的 DPD 介观 模拟方法，运用粗粒化模型，用单个珠子代表多个原子 组成的基团, 各珠子由弹簧简谐振子连接, 并通过保守 力、耗散力和随机力三种作用力描述珠子所受作用力, 依据牛顿运动方程利用柔性势能函数描述珠子的运动, 通过求解一系列的运动方程得到珠子的运动轨迹 ${ }^{[26,27]}$. 在模拟过程中，珠子的质量、距离、时间和温度都采用 $\mathrm{DPD}$ 单位, 能量采用 $k_{B} T$ 为单位 ${ }^{[28]}$. 各珠子间的相互作 用由珠子间的相互作用参数 $a$ 来表征:

$$
\begin{aligned}
& a_{i i}=75 k_{B} T / \rho \\
& a_{i j}=a_{i i}+3.27 \chi_{i j}(T), \quad \rho=3
\end{aligned}
$$

$\rho$ 为珠子密度, $\chi_{i j}$ 通过 Blends 模块进行全原子模拟计算 珠子 $i, j$ 之间的相互作用混合能得到.

模拟中采用 $20 \times 10 \times 10$ 具有周期性边界条件的格 子 ${ }^{[29]}$, 噪声振幅选择 $\sigma=3$, 弹性常数 4.0. 体系模拟运 行 20000 步, 步长 0.05 , 模拟体系通过 Gibbs 正则系综 达到平衡.

\section{3 .3 分子动力学模拟}

已有文献 ${ }^{[24]}$ 报道，采用双层膜建立模拟模型，采用 六方密堆积的方式排列表面活性剂分子，获得的分子模 拟结果与实验结果对应很好, 故采用此种建模方式, 并 根据需要适当改变分子个数建立模拟体系的初始模型.

水分子采用 $\mathrm{SPC}-\mathrm{E}^{[30]}$ 模型，水盒子大小与表面活性 剂层的盒子一致，表面活性剂的反离子在建模时随机加 入. 对于含无机盐的水溶液, 无机离子在建立水盒子时 一起加入. 水的密度根据具体的模拟温度相应改变.

对泡沫液膜的模拟采用 “三明治” 模型 ${ }^{[31]}$. 最终模 型的 $\mathrm{Z}$ 方向长度足够长以避免周期性边界盒子之间相 互作用. 对初始模型运行最小化 20000 步，去除初始模 型可能存在的分子重叠 ${ }^{[32]}$. 
模拟采用 NVT 系综, Velocity-Verlet ${ }^{[33]}$ 积分方法, 积 分步长为 1.0 fs. Nose-Hoover ${ }^{[34,35]}$ 热浴方法控制温度, 耦合常数为 $0.1 \mathrm{ps}$. 运用分子动力学模拟使体系达到平 衡, 平衡后的结果用于分析.

采用(2)式计算界面生成能(Interfacial Formation Energy, IFE), 用以考察表面活性剂插入到界面上对体 系能量的影响:

$$
\operatorname{IFE}=\left(E_{\text {interface }}-E_{\text {solution }}\right) / n
$$

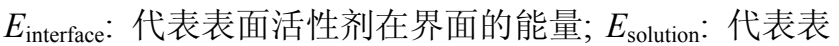
面活性剂在体相溶液中的能量; $n$ : 表面活性剂总的个 数.

\section{References}

[1] Jing, Z.-S. Overview of Surface Active Agent, China Light Industry Press, Beijing, 1999, pp. 191 242. (荆忠胜, 表面活性剂概论, 中 国轻工业出版社, 北京, 1999, pp. 191 242.)

[2] Malysa, K. Adv. Colloid Interface Sci. 1992, 40(2), 37.

[3] Sánchez, C. C.; Patino, J. M. R. Food Hydrocolloids 2005, 19(3), 407.

[4] Farajzadeh, R.; Andrianov, A.; Zitha, P. L. J. Ind. Eng. Chem. Res. 2010, 49(4), 1910

[5] Yan, Y.-L.; Zhang, N.-S.; Qu, C.-T.; Liu, L.; Gao, Y.-L. Acta Chim. Sinica 2006, 64(1)，54. (燕永利, 张宁生, 屈撑囤, 刘立, 高永利, 化学学报, 2006, 64(1), 54.)

[6] Li, Y.; He, X.-J.; Cao, X.-L.; Zhao, G.-Q.; Tian, X.-X.; Cui, X.-H. J. Colloid Interface Sci. 2007, 307, 215.

[7] Acharya, D. P.; Gutiérrez, J. M.; Aramaki, K.; Aratani, K.; Kunieda, H. J. Colloid Interface Sci. 2005, 291, 236.

[8] Jiang, Y.; Lu, X.-Y.; Chen, H.; Mao, S.-Z.; Liu, M.-L.; Luo, P.-Y.; Du, Y.-R. J. Phys. Chem. B 2009, 113, 8357.

[9] Szymczyk, K.; Jańczuk, B. J. Colloid Interface Sci. 2006, 303, 319.

[10] Dar, A. A.; Chatterjee, B.; Rather, G. M.; Das, A. R. J. Colloid Interface Sci. 2006, 298, 395.

[11] Shiloach, A.; Blankschtein, D. Langmuir 1998, 14(25), 7166.

[12] Hines, J. D.; Thomas, R. K.; Garrett, P. R.; Rennie, G. K. J. Phys. Chem. B 1998, 102, 8834 .

[13] Prajapati, R. R.; Bhagwat, S. S. J. Chem. Eng. Data 2012, 57, 3644.
[14] Danov, K. D.; Kralchevska, S. D.; Kralchevsky, P. A.; Ananthapadmanabhan, K. P.; Lips, A. Langmuir 2004, 20, 5445.

[15] Zhang, Z.-Q.; Xu, G.-Y.; Ye, F.; Zheng, L.-Q.; Luan, Y.-X. Acta Phys.-Chim. Sinica 2001, 17(12), 1122. (张志庆, 徐桂英, 叶繁, 郑立强, 奕玉霞, 物理化学学报, 2001, 17(12), 1122.)

[16] Srinivasan, V.; Blankschtein, D. Langmuir 2003, 19, 9946.

[17] Fang, Y.; Xia, Y.-M. China Surfactant Detergent \& Cosmetics 1998 (6), 22. (方云, 夏咏梅, 日用化学工业, 1998, (6), 22.)

[18] Xia, J.-D.; Fang, Y.; Zhu, S.-P. The Fourth International Surfactants Congress and Exhibition, Barcelona, 1996(C), 72.

[19] Li, Y.; He, X.-J.; Cao, X.-L.; Shao,Y.-H.; Li, Z.-Q.; Dong, F.-L. Mol. Simul. 2005, 31(15), 1027.

[20] Hu, X.-Y.; Li, Y.; He, X.-J.; Li, C.-X.; Li, Z.-Q.; Cao, X.-L.; Xin, X.; Somasundaran, P. J. Phys. Chem. B 2012, 116, 160.

[21] Koehler, S. A.; Hilgenfeldt, S.; Stone, H. A. Langmuir 2000, 16, 6327.

[22] Hu, X.-Y.; Song, X.-W.; Li, Q.-W.; He, X.-J.; Wang, Q.-W.; Li, Y Acta Chim. Sinica 2009, 67(14), 1691. (胡晓莹, 宋新旺, 李全伟, 何秀娟, 王其伟, 李英, 化学学报, 2009, 67(14), 1691.)

[23] Bhattacharyya, A.; Monroy, F.; Langevin, D.; Argillier, J. F. Langmuir 2000, 16, 8727.

[24] Li, C.-X.; Li, Y.; Yuan, R.; Lv, W.-Q. Langmuir 2013, $29(18), 5418$.

[25] Hoogerbrugge, P. J.; Koelman, J. M. V. A. Europhys. Lett. 1992, 19, 155.

[26] Rekvig, L.; Kranenburg, M.; Vreede, J.; Hafskjold, B.; Smit, B Langmuir 2003, 19(20), 8195 .

[27] Zhu, P.-F.; Li, Y.; Li, Q.-W.; Song, X.-W.; Cao, X.-L.; Li, Z.-Q. Acta Chim. Sinica 2011, 69(20), 2420. (朱鹏飞, 李英，李全伟，宋新旺， 曹绪龙, 李振泉, 化学学报, 2011, 69(20), 2420.)

[28] Li, Z.-Q.; He, X.-J.; Li, Y.; Ma, B.-M.; Cao, X.-L.; Song, X.-W.; Cui, X.-H. Acta Chim. Sinica 2007, 65(24), 2803. (李振泉, 何秀娟, 李英，马保民，曹绪龙，宋新旺，崔晓红，化学学报, 2007, 65(24), 2803.)

[29] Dong, F.-L.; Li, Y.; Zhang, P. Chem. Phys. Lett. 2004, 399, 215.

[30] Berendsen, H. J. C.; Grigera, J. R.; Straatsma, T. P. J. Phys. Chem. 1987, 91, 6269 .

[31] Gamba, Z.; Hautman, J.; Shelley, J. C.; Klein, M. L. Langmuir 1992 8,3155 .

[32] Hu, X.-Y.; Li, Y.; Zhang, H.; He, X.-J.; Xue, Y.-Z.; Wang, P.; Yang, J.-L. Acta Chim. Sinica 2010, 68(2), 131. (胡晓莹, 李英, 张辉, 何 秀娟, 薛玉志, 王萍, 杨景利, 化学学报, 2010, 68(2), 131.)

[33] Verlet, L. Phys. Rev. 1967, 159, 98.

[34] Nose, S. J. Chem. Phys. 1984, 81, 511.

[35] Hoover, W. G. Phys. Rev. A 1985, 31, 1695. 